This item was submitted to Loughborough's Research Repository by the author.

Items in Figshare are protected by copyright, with all rights reserved, unless otherwise indicated.

\title{
Adaptive phase-shifting algorithm for temporal phase evaluation
}

PLEASE CITE THE PUBLISHED VERSION

PUBLISHER

(C) Optical Society of America

VERSION

AM (Accepted Manuscript)

LICENCE

CC BY-NC-ND 4.0

REPOSITORY RECORD

Ruiz, Pablo D., Jonathan M. Huntley, and Guillermo H. Kaufmann. 2019. "Adaptive Phase-shifting Algorithm for Temporal Phase Evaluation”. figshare. https://hdl.handle.net/2134/5930. 
This item was submitted to Loughborough's Institutional Repository (https://dspace.lboro.ac.uk/) by the author and is made available under the following Creative Commons Licence conditions.

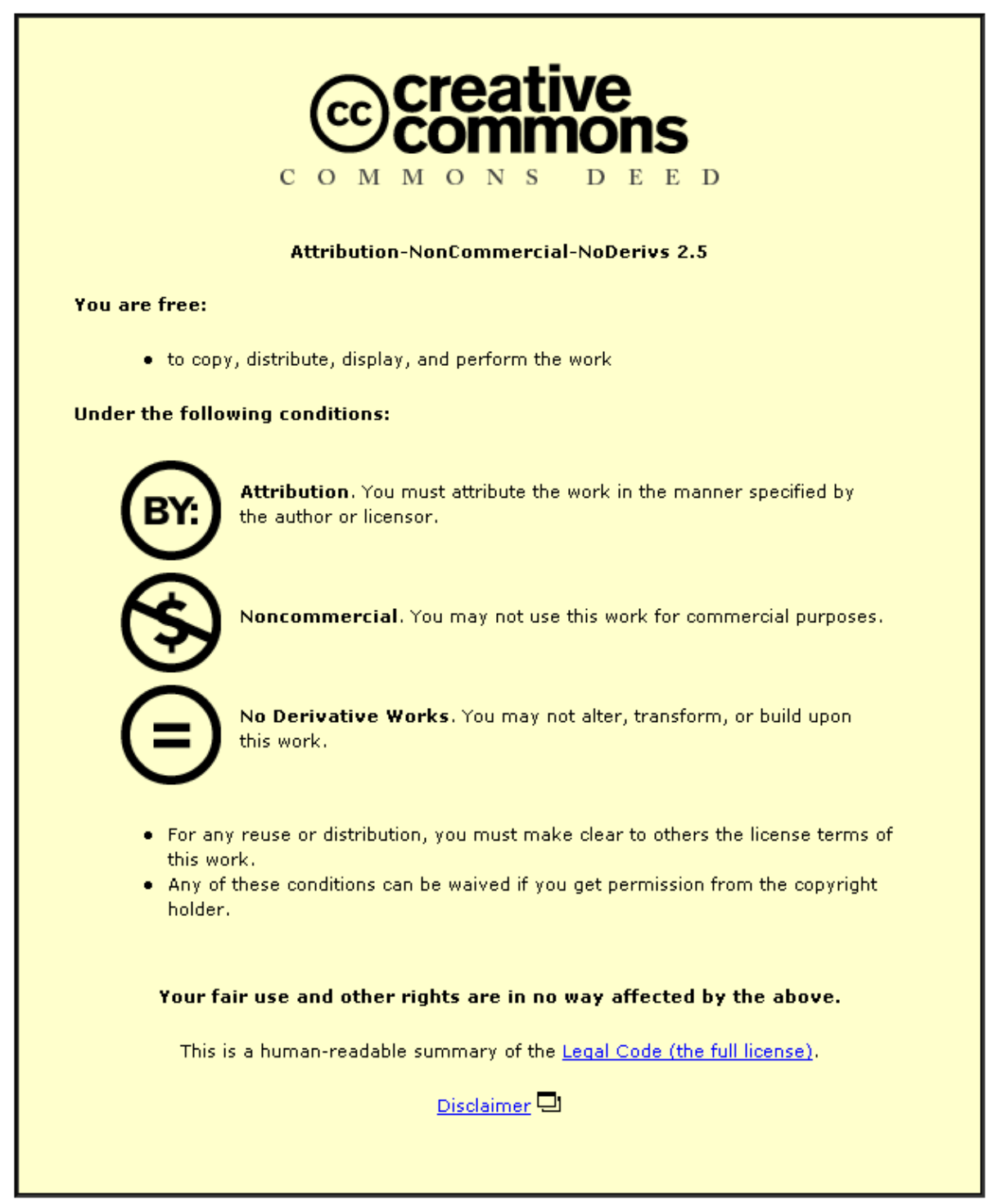

For the full text of this licence, please go to: http://creativecommons.org/licenses/by-nc-nd/2.5/ 


\title{
Adaptive phase-shifting algorithm for temporal phase
}

\author{
evaluation
}

\author{
Pablo D. Ruiz
}

Instituto de Física Rosario, Bvd. 27 de Febrero 210 bis, S2000EZP Rosario, Argentina e-mail: P.D.Ruiz@,lboro.ac.uk

\section{Jonathan M. Huntley}

Department of Mechanical Engineering, Loughborough University, Loughborough, Leics. LE11 3TU, UK

e-mail: J.M.Huntley@,lboro.ac.uk

\section{Guillermo H. Kaufmann}

Instituto de Física Rosario and Departamento de Física, Facultad de Ciencias Exactas, Ingeniería y Agrimensura, Universidad Nacional de Rosario, Bvd. 27 de Febrero 210 bis, S2000EZP Rosario, Argentina e-mail: guille@ifir.edu.ar 


\begin{abstract}
Most standard temporal phase-shifting algorithms evaluate the phase by computing a windowed Fourier transform (WFT) of the intensity signal at the carrier frequency of the system. However, displacement of the specimen during image acquisition may cause the peak of the transform to shift away from the carrier frequency, leading to phase errors and even unwrapping failure. We present a novel TPS method that searches for the peak of the WFT and evaluates the phase at that frequency instead of at the carrier frequency. The performance of this method is compared with that of standard algorithms by using numerical simulations. Experimental results from highspeed speckle interferometry studies of carbon fiber panels are also presented.
\end{abstract}

OCIS codes: $120.6160,120.5050,120.2650,120.7280,120.4290$. 


\section{Introduction}

During the last two decades, Electronic Speckle Pattern Interferometry (ESPI) has become a standard technique for obtaining accurate, reproducible and fast measurements of shape and deformation of rough surface objects. ${ }^{1}$ The high sensitivity of this technique constitutes at the same time both its main strength and its main drawback, especially when it is aimed to be applicable in real world environments, because of its vulnerability to disturbances such as thermal convection and vibration. ${ }^{2}$ Partial solutions to this problem have been developed such as the use of pulsed lasers, active phase stabilisation systems, ${ }^{3}$ shearing interferometers and dynamic high-speed phase-shifting ESPI systems. ${ }^{4-7}$ This last approach is a rapidly growing one due to the lower cost of high-speed image sensors, the availability of fast phase modulators, and the important advantages that temporal phase-shifting (TPS) methods present when combined with temporal phase unwrapping.

When a coherent beam propagates from a test object and interferes with a temporal phaseshifted reference beam on the image plane of an optical system, a modulated intensity signal is obtained at each pixel. If this intensity distribution is sampled at a temporal rate high enough to satisfy the Nyquist condition, then the series of images can be evaluated with TPS algorithms to compute the object wrapped phase evolution at any given pixel. ${ }^{2}$ This procedure is carried out through the evaluation of the arctangent function of a ratio of two linear combinations of a set of measured intensities. Afterwards, the phase change relative to the first phase state can be obtained by means of temporal unwrapping. ${ }^{8}$ The physical measurand of interest (e.g. shape, deformation, displacement or index of refraction fields) is finally computed from the phase distributions and the knowledge of the sensitivity vector of the interferometer. 
TPS algorithms are susceptible to random and systematic error sources such as higher harmonics in the intensity signal, phase shifter miscalibration, nonlinear response of the photodetectors, random intensity noise, speckle decorrelation and vibration. Thus, they have been developed to obtain error-free phase distributions, at least up to a second order dependence on the error parameters. ${ }^{4,9-12}$

When a dynamic high-speed TPS interferometer is used off the optical table, it is likely to be subjected to environmental vibration, for example when relatively low strain-rate tests are carried out on materials or structures using loading by vacuum or by tensile test machine. The effect of vibration on the unwrapped phase depends on the particular phase shifting algorithm used, the amplitude and frequency content of the vibration and the object phase itself. The object phase dependence introduces a phase error that appears as a ripple with twice the spatial frequency of the original fringe pattern in smooth wavefront interferometers or as a random field in speckle interferometers. In order to obtain a successful measurement under such conditions, two requirements must be fulfilled during image acquisition. (i) The total phase change between successive images resulting from the deformation, the vibration, and the phase shifter, must lie within the range $(-\pi, \pi)$ at all points on the object. The upper limit is equivalent to requiring that the temporal intensity signal for each pixel is sampled according to the Nyquist condition, which depends on the framing rate of the camera, the phase step between samples and the object displacement (deformation plus vibration). Likewise, the lower limit follows from the need to avoid interference from the dc and negative frequency peaks of the spectrum. (ii) The object motion and the acquisition sampling rate must be such that the time evolution of the observed displacements can be approximated reasonably well by its first-order Taylor 
polynomial expansion, i.e. the displacement is approximately a linear function of time. Such a case corresponds to linear miscalibration of the phase shifter. Many modern TPS algorithms are insensitive to linear errors but not to higher order miscalibration errors.

In addition, the particular TPS algorithm used for phase evaluation should: (iii) be insensitive to high-frequency vibration components in order to obtain a phase distribution with low rms spatial error, and (iv) be able to keep track of high-amplitude, low-frequency vibration without losing the continuity of the measurement.

Previous research on harmonic and random vibration showed that standard TPS algorithms cannot fulfill requirements (iii) and (iv) at the same time. ${ }^{13,14}$ Long window TPS algorithms present low sensitivity to high-frequency vibration, but they are vulnerable to highamplitude low-frequency vibration. In Ref. [14] it was suggested that an adaptive TPS algorithm could be used to evaluate the object phase when using long window TPS algorithms in the presence of low-frequency vibration.

Of the many TPS algorithms published to date, only a small fraction can be considered to be adaptive. Of these, the four-frame Carré algorithm is the best known. ${ }^{15} \mathrm{~A}$ five-frame formula following the same idea was described by de Lega. ${ }^{4}$ A more general adaptive algorithm, based on searching the phase of a wavelet transform of the intensity signal was proposed in Refs. [4] and [16]. Although there are similarities between the wavelet transform and windowed Fourier transform (WFT) used here, one drawback of the wavelet transform should perhaps be pointed out, namely that the temporal and frequency resolution is different for sample velocities having equal magnitude but opposite sign.

Our purpose in this paper is to present the results of a TPS algorithm that searches for the peak in the modulus of the windowed Fourier transform. Numerical simulations were carried out representing object motion in the presence of pseudo random vibration with 
known spectral content. Throughout this paper, we regard the vibration as a source of noise whose influence we are trying to minimise by adaptive phase shifting evaluation. The performance of several standard and adaptive TPS algorithms was studied and compared by evaluating the rms phase change error and the unwrapping success rate. A theoretical description of the ridge-searching algorithm is presented in Section 2. Numerical results and discussions are presented respectively in Sections 3 and 4. Experimental confirmation of the simulated results is presented in Section 5, using data from the $1 \mathrm{kHz}$ phase-shifting speckle interferometer described in Ref. 5, with conclusions summarized in Section 6.

\section{Ridge Searching Algorithm}

In this section we present the ridge searching algorithm that is used to improve the phase estimation obtained through standard TPS algorithms. We assume the use of an out-ofplane interferometer like the one shown in Fig. 4; other interferometer configurations require simply a different scaling factor relating phase change to displacement. For completeness and clarity we include a transcript of the equations for phase evaluation, Eq. (1) to Eq. (5), that may be found in Refs. [1] (chapter 2), [5], [13] and [14].

A set of $N_{\mathrm{t}}$ intensity samples recorded by a single pixel in a temporal phase-shifting interferometer can be written as:

$$
I(t)=I_{0}+I_{m} \cos (\Phi+\phi t) t=0,1, \ldots, N_{\mathrm{t}}-1
$$

where $I_{0}$ is the de term of the signal, $I_{m}$ is the amplitude of the intensity modulation, $\Phi=\Phi_{\mathrm{s}}$

$+\Phi_{\mathrm{d}}$ is the object phase to be measured which includes a speckle random phase $\Phi_{\mathrm{s}}$ and a phase term $\Phi_{\mathrm{d}}$ due to object displacement, $\phi=2 \pi / N$ is the phase shift between successive samples that in our interferometer was introduced by a Pockels cell ( $N$ being an integer 
number of samples per $2 \pi$ cycle of the carrier) and $t$ is a non-dimensional temporal index or frame number.

A wrapped $(\bmod 2 \pi)$ estimator of the object phase change between frame $t$ and the first frame in the sequence, $t=0$, is given by:

$$
\Delta \hat{\Phi}_{w}(t, 0)=\tan ^{-1}\left[\frac{N(t) D(0)-D(t) N(0)}{D(t) D(0)+N(t) N(0)}\right], \quad t=0,1, \ldots, N_{t}-M
$$

where $N(t)$ and $D(t)$ are respectively proportional to $\sin \Phi(t)$ and $\cos \Phi(t)$, and are given by

$$
N(t)=\operatorname{Im}[Z(t)], \quad D(t)=\operatorname{Re}[Z(t)],
$$

and where $N_{t}$ is the total number of frames in the sequence. Phase changes are calculated in order to remove the initial random speckle phase distribution $\Phi_{\mathrm{s}}$, which is assumed to remain constant. The complex variable $Z(t)$ is defined as:

$$
Z(t)=\left[\sum_{t^{\prime}=0}^{M-1} a\left(t^{\prime}\right) I\left(t+t^{\prime}\right)+i \sum_{t^{\prime}=0}^{M-1} b\left(t^{\prime}\right) I\left(t+t^{\prime}\right)\right] \exp (-i \phi t),
$$

where the coefficients $a(t)$ and $b(t)$ depend on the particular phase shifting algorithm used to calculate $\Delta \hat{\Phi}_{w}(t, 0)$. The complex exponential term in Eq. (4) eliminates the signal modulation introduced by the temporal carrier phase $\phi t=2 \pi t / N$.

By defining

$$
a\left(t^{\prime}\right)+i b\left(t^{\prime}\right)=w\left(t^{\prime}\right) \exp \left(-i \phi t^{\prime}\right), \quad t^{\prime}=0,1,2, \ldots, M-1,
$$


it can be seen that the term in the square brackets in Eq. (4) is equivalent to the discrete windowed Fourier transform (WFT) with respect to the time variable $t$, of the intensity signal evaluated at the modulation frequency $k_{t}=1$ (see Ref. [1] (chapter 2)):

$$
\widetilde{I}\left(k_{t}, t\right)=\sum_{t^{\prime}=0}^{M-1} w\left(t^{\prime}\right) I\left(t+t^{\prime}\right) \exp \left(-i 2 \pi k_{t} t^{\prime} / N\right)
$$

where $w$ is a sampling window of length $M$, and the notation $\widetilde{I}$ denotes the Fourier transform of $I$. A particular case of the window function $w(t)$ is the well-known Hanning window,

$$
w(t)=\frac{1}{2}+\frac{1}{2} \cos \left\{\frac{2 \pi}{M}\left[t-\left(\frac{M-1}{2}\right)\right]\right\} \quad t=0,1, \ldots, M-2
$$

Equation (6) can be expressed in terms of the continuous Fourier transform as:

$$
\widetilde{I}\left(k_{t}, t\right)=\int_{-\infty}^{\infty} w(\tau) I(t+\tau) \sum_{n=-\infty}^{\infty} \delta(\tau-n) \exp \left(-i 2 \pi k_{t} \tau / N\right) d \tau
$$

where $\tau$ is a continuous non-dimensional variable, $n$ is an integer number and $\delta()$ is the Dirac delta function. The right hand side of Eq. (8) can be interpreted as the Fourier transform of a sampled intensity distribution $I(t+\tau) \sum_{n=-\infty}^{\infty} \delta(\tau-n)$ multiplied by a continuous window function $w(\tau)$. The product $w(\tau) I(t+\tau) \sum_{n=-\infty}^{\infty} \delta(\tau-n)$ is then equal to the discrete set $w\left(t^{\prime}\right) I\left(t+t^{\prime}\right)$ with $t^{\prime}=0,1, \ldots, M-1$. 
Using Eq. (1) evaluated at time $t+\tau, I(t+\tau)=I_{0}+I_{m} \cos (\Phi+\phi t+\phi \tau)$, making use of the convolution theorem, and the fact that $\cos \theta=[\exp (i \theta)+\exp (-i \theta)] / 2$, the Fourier transform in Eq. (8) can be worked out to obtain:

$\widetilde{I}\left(k_{t}, t\right)=\widetilde{w}\left(k_{t}\right) *\left[A\left(k_{t}\right)+B_{+}\left(k_{t}, t\right)+B_{-}\left(k_{t}, t\right)\right]$

where

$$
\begin{aligned}
& A\left(k_{t}\right)=I_{0} \sum_{j=-\infty}^{\infty} \delta\left(k_{t}-j N\right) \\
& B_{+}\left(k_{t}, t\right)=\frac{I_{m}}{2} \exp (i \phi t) \int_{-\infty}^{\infty} \exp (i \Phi) \exp \left(-i 2 \pi \tau\left(k_{t}-1\right) / N\right) \sum_{n=-\infty}^{\infty} \delta(\tau-n) d \tau \\
& B_{-}\left(k_{t}, t\right)=\frac{I_{m}}{2} \exp (-i \phi t) \int_{-\infty}^{\infty} \exp (-i \Phi) \exp \left(-i 2 \pi \tau\left(k_{t}+1\right) / N\right) \sum_{n=-\infty}^{\infty} \delta(\tau-n) d \tau .
\end{aligned}
$$

and where $*$ indicates the convolution operation. The three terms occur respectively at $k_{t}=$ $0,+1$ and -1 and represent the continuous Fourier transform of a cosine wave plus a dc offset. These peaks are repeated along the $k_{t}$ axis at integral multiples of $N$ due to the sampling of the original signal. In Eq. (9), the convolution with the transform of the window function is equivalent to placing a copy of $\widetilde{w}\left(k_{t}\right)$ centered on each delta function. A schematic view of the spectrum is shown in Fig. 1. 
If the object phase $\Phi=\Phi_{\mathrm{s}}+\Phi_{\mathrm{d}}$ remains constant during the acquisition of the $M$ frames, the terms $\exp ( \pm i \Phi)$ can be taken out of the integrals. Then, for $j=0, \widetilde{I}\left(k_{t}, t\right)$ is equal to the discrete WFT of $I(t)$ and:

$$
\begin{aligned}
& A\left(k_{t}\right)=I_{0} \delta\left(k_{t}\right), \\
& B_{+}\left(k_{t}, t\right)=\frac{I_{m}}{2} \exp [i(\Phi+\phi t)] \delta\left(k_{t}-1\right), \\
& B_{-}\left(k_{t}, t\right)=\frac{I_{m}}{2} \exp [-i(\Phi+\phi t)] \delta\left(k_{t}+1\right) .
\end{aligned}
$$

In general, $I_{0}, I_{\mathrm{m}}$, and $\Phi=\Phi_{\mathrm{s}}+\Phi_{\mathrm{d}}$ in Eq. (1) are all functions of time. We will assume a negligible variation of $I_{0}, I_{\mathrm{m}}$ and $\Phi_{\mathrm{s}}$ but a slow evolution of the object phase $\Phi_{\mathrm{d}}$ during the acquisition of the $M$ frames. In this case, $\Phi_{\mathrm{d}}(\mathrm{t})$ can be approximated by a first order Taylor expansion:

$$
\Phi_{d}(t+\delta t) \cong \Phi_{d}(t)+\dot{\Phi}_{d}(t) \delta t
$$

where $-\Delta t \leq \delta t \leq \Delta t$ and $\Delta t=M / 2$ is the half-width of the sampling window, $\Phi_{d}(t)$ is a phase term which is constant during frame acquisition and $\dot{\Phi}_{d}(t)$ is the object phase temporal gradient (in radians $\times$ frame ${ }^{-1}$ ), which is proportional to the out-of-plane component of velocity $\dot{z}(t)$ of the sample: 


$$
\dot{\Phi}_{d}(t)=\frac{4 \pi}{\lambda} \dot{z}(t)
$$

Standard TPS algorithms evaluate the phase from $M$ frames starting at time $t$ and the value obtained is assigned to the frame $t+M / 2 .{ }^{14}$ In Eq. (16), the phase is also computed at $t+$ $\Delta t$.

Considering Eqs. (11), (12) and (16), the terms $\mathrm{B}_{+}$and $\mathrm{B}_{\text {- close to the normalized frequency }}$ axis origin, $k_{t}=0$, will be given by:

$$
\begin{aligned}
& B_{+}\left(k_{t}, t\right)=\frac{I_{m}}{2} \exp \left\{i\left[\Phi_{s}+\Phi_{d}(t)+\phi t\right]\right\} \delta\left[k_{t}-\left(1+\frac{\dot{\Phi}_{d}(t) N}{2 \pi}\right)\right], \\
& B_{-}\left(k_{t}, t\right)=\frac{I_{m}}{2} \exp \left\{-i\left[\Phi_{s}+\Phi_{d}(t)+\phi t\right]\right\} \delta\left[k_{t}+\left(1+\frac{\dot{\Phi}_{d}(t) N}{2 \pi}\right)\right] .
\end{aligned}
$$

It can be seen that the effect of the phase gradient $\dot{\Phi}_{d}(t)$ is to shift the normalized carrier frequency from $k_{t}=1$ to the normalized ridge frequency

$$
k_{r}(t)=1+\frac{\dot{\Phi}_{d}(t) N}{2 \pi}
$$

This effect is shown schematically in Fig. 1. The phase $\Phi_{s}+\Phi_{d}(t)$ can now be computed at the first order approximation of the instantaneous carrier frequency $k_{r}(t)$ instead of at $k_{t}=1$. In order to do this, it is necessary to find $k_{r}(t)$, which by definition is the maximum magnitude of the WFT for time $t$, excluding the dc peak. Computing this maximum for all $t$, the ridge of the temporal signal is extracted. Afterwards, the WFT is evaluated over $k_{r}(t)$ using Eqs. (9), (18) and (19): 


$$
\begin{aligned}
\widetilde{I}\left[k_{r}(t), t\right]= & \widetilde{w}\left[k_{r}(t)\right] I_{0}+ \\
& \frac{I_{m}}{2} \widetilde{w}(0) \exp \left\{i\left[\Phi_{s}+\Phi_{d}(t)+\phi t\right]\right\}+ \\
& \frac{I_{m}}{2} \widetilde{w}\left[2 k_{r}(t)\right] \exp \left\{i\left[\Phi_{s}+\Phi_{d}(t)+\phi t\right]\right\}
\end{aligned}
$$

For a Hanning window with $M$ samples, the first minimum occurs at $k_{t}=8 / M$, that is at 0.5 , 0.25 , and 0.125 for $M=16,32$ and 64 respectively, with subsidiary peaks below $0.023 \widetilde{w}(0)$. Therefore, as $\widetilde{w}\left(k_{r}\right)<0.023 \widetilde{w}(0)$ for $k_{r}>8 / M$, the first and third terms in Eq. (21) can be neglected. In this case, Eq. (21) is reduced to:

$$
\widetilde{I}\left[k_{r}(t), t\right]=\frac{I_{m}}{2} \widetilde{w}(0) \exp \left\{i\left[\Phi_{s}+\Phi_{d}(t)+\phi t\right]\right\}
$$

The zero order approximation of the wrapped object phase change, $\Delta \hat{\Phi}_{d w}(t, 0)$, can then be obtained from Eqs. (2) and (3) using:

$$
Z(t)=\widetilde{I}\left[k_{r}(t), t\right] \exp (-i \phi t)
$$

Using Eq. (16), together with the relationship between the phase gradient $\dot{\Phi}_{d}$ and the ridge normalized frequency specified by Eq. (20) for $N=4$ :

$$
\dot{\Phi}_{d}(t)=\frac{\pi}{2}\left[k_{r}(t)-1\right]
$$

together with the fact that $\Delta t=M / 2$, the object wrapped phase change approximated to the first order at the centre of the time window is evaluated by:

$$
\Delta \hat{\Phi}_{d w}\left(t+\frac{M}{2}, 0\right) \cong \Delta \hat{\Phi}_{d w}(t, 0)+\frac{\pi M}{4}\left[k_{r}(t)-1\right]
$$


Finally, the continuous phase change $\Delta \hat{\Phi}_{d u}(t+\Delta t, 0)$ is obtained through temporal phase unwrapping using the method described in Ref. [5] as algorithm 2.

The ridge-searching algorithm can be summarized as follows:

1. Set $t=0$.

2. Subtract de term from $I\left(t+t^{\prime}\right)$, with $t^{\prime}=0,1, \ldots, \mathrm{M}-1$.

3. Evaluate $\widetilde{I}\left[k_{r}(t), t\right]$ using Eq. (6) and the intensity samples obtained in step 2.

4. Compute the value $k_{r}(t)$ for which $\left|\widetilde{I}\left[k_{r}(t), t\right]\right|$ is a maximum by means of a root searching algorithm such as binary chop, Newton-Raphson, etc.

5. Evaluate the zero order approximation of the object phase change $\Delta \hat{\Phi}_{d w}(t, 0)$ using Eq. (23).

6. Evaluate the first order approximation of the object phase change $\Delta \hat{\Phi}_{d w}(t+\Delta t, 0)$ using Eq. (25).

7. Unwrap $\Delta \hat{\Phi}_{d w}(t+\Delta t, 0)$.

8. Set $t=t+1$ and repeat steps 2 to 8 until $t=N_{t}-\mathrm{M}$.

\section{Numerical Simulation}

\section{A Pseudo-Random Vibrations}

In order to evaluate the performance of the ridge-searching algorithm, the intensity signal at an arbitrary pixel was simulated using the method described in Ref. [13]. The simulations represented the interference signal between a temporal phase-shifted reference beam with $\phi$ $=\pi / 2$, and an object beam propagating from a surface submitted to pseudo-random 
vibration. Normally, the spectral content of vibration is specified in terms of target velocity. Design criteria for metrology laboratories are often based on either peak velocity spectra or probability density spectra for velocity. These are typically specified to be flat from dc up to a corner frequency, $f_{0}$, with a high frequency roll-off. ${ }^{17,18}$ This approach was adopted in the present study by specifying a spectral density function for velocity, $S(f)$ as follows:

$$
S(f)=\left\{\begin{array}{lr}
S_{0} & 0<f \leq f_{0} \\
\frac{S_{0} f_{0}}{f} & f_{0}<f
\end{array}\right.
$$

where $S_{0}$ is the spectral density for the flat portion of the spectrum (with units of $\mu \mathrm{m}^{2} \mathrm{~s}^{-1}$ ). This function has a $20 \mathrm{~dB}$ per decade roll-off above the corner frequency $f_{0}$, the value of which was fixed at $50 \mathrm{~Hz}$. The severity of the vibration was controlled by varying the single parameter $S_{0}$.

The desired spectral content was achieved by frequency-domain filtering a one-dimensional array of independent random numbers from a normal distribution with zero mean and unit variance. A $20 \mathrm{~dB} / \mathrm{dec}$ rolloff filter was applied to its amplitude spectrum from DC to the corner frequency $f_{0}$, and another $20 \mathrm{~dB} / \mathrm{dec}$ rolloff above $f_{0}$. The real part of the inverse Fourier transform of the filtered spectrum, after suitable scaling, then specified the sample displacement time history $z(t)$. Figure 2 is an example of an average of 100 independent velocity spectra created in this way with a scaling chosen such that the rms phase amplitude was $\sigma_{\phi}=2 \pi$ and the corresponding rms displacement amplitude was $\sigma_{z}=\lambda / 2$. The spectrum is seen to follow the form specified by Eq. (26), with a mean spectral density at the plateau, $S_{0}=0.51$

\section{B Rms Phase Change Error}


Simulations were carried out in accordance with the procedure described in Ref. 14. In a speckle interferometer the object phase $\Phi$ includes an implicit speckle phase term $\Phi_{\mathrm{s}}$ that varies from point to point in space and that is uniformly distributed over the range $[-\pi, \pi)$.

This spatial variation causes the errors in the unwrapped phase change values $\Delta \hat{\Phi}_{u}$ to vary from one pixel to another. In the simulations, therefore, $L$ object phase values $\Phi_{l}$ have been chosen equally spaced over the range $[-\pi, \pi)$ and the index $l$ was used to designate them. Moreover, any frame $t_{j}$ can be considered as the reference frame for the remaining ones. Therefore, the rms phase change error $\sigma\left(t, t_{j}\right)$ for a given time $t$ and reference frame $t_{j}$ has to be averaged over all possible object phase values $\Phi_{l}$. This can be written as:

$$
\sigma\left(t, t_{j}\right)=\left\{\frac{1}{L} \sum_{l=0}^{L-1}\left[\Delta \hat{\Phi}_{u}\left(t, t_{j}, l\right)-\left\langle\Delta \hat{\Phi}_{u}\left(t, t_{j}\right)\right\rangle\right]^{2}\right\}^{1 / 2},
$$

with

$$
\left\langle\Delta \hat{\Phi}_{u}\left(t, t_{j}\right)\right\rangle=\frac{1}{L} \sum_{l=0}^{L-1} \Delta \hat{\Phi}_{u}\left(t, t_{j}, l\right)
$$

$\sigma\left(t, t_{j}\right)$ measures the dispersion of the phase change obtained for different pixels, varies rapidly in time and depends on the reference frame chosen. After averaging over these indexes, we obtain:

$$
\bar{\sigma}=\frac{1}{s \cdot s_{j}} \sum_{t=1}^{s} \sum_{t_{j}=1}^{s_{j}} \sigma\left(t, t_{j}\right)
$$


where $s_{j}$ is the number of reference frames considered and $s=N_{\mathrm{t}}-M$ is the maximum $t$ value for which a phase value can be calculated. In this way, $\bar{\sigma}$ represents an average rms measure of the spatial dispersion in calculated phase change values.

\section{Unwrapping success rate}

When measurements are carried out in the presence of vibration, phase errors will appear as described in the previous section, but the most damaging effect is the possible inclusion of a $2 \pi$ unwrapping error in the estimated phase. This error depends on the presence of steep gradients in the input phase as well as the amplitude of modulation of the intensity signal and the particular TPS algorithm used. Thus, in order to evaluate the probability of a successful phase evaluation for a given TPS algorithm, we computed the unwrapping success rate (USR). This parameter is defined as the ratio of the number of successfully unwrapped signals to the total number of unwrapped ones. The unwrapping of a wrapped phase time sequence of given length is assumed to be correct if the unwrapped phase agrees with the corresponding input phase to within $\pm \pi$ for the last frame of the sequence. Even though this condition may be fulfilled for the last frame, large phase gradients can cause the unwrapped and the input phases to split apart and to match again by chance during some time within the sequence. Though this case should not be considered strictly as a successful unwrapping, it is quite rare, at least for the cases of interest where the probability of a successful unwrapping is close to 1 . The unwrapping success rate does not refer to the unwrapping process alone, but mainly to the phase evaluation procedure. The numerical simulation allows us to evaluate this parameter for several conditions that cannot be carried out experimentally and also to associate the cause of failure to the vibration itself and not to other sources of error. 


\section{Results and Discussion}

When $k_{r}=1$, Eq. (25) reduces to the phase estimator normally used when applying standard TPS algorithms. In this case, $\Delta \hat{\Phi}_{d w}(t+\Delta t, 0)$ will be equal to the zero order term $\Delta \hat{\Phi}_{d}(t, 0)$ and corresponds to the case where the object is motionless. However, displacement of the object during acquisition or variations in the phase steps introduced by the phase-shifter device may cause the peak of the transform to shift away from $k_{t}=1$. While the peak shift is proportional to the object velocity in the direction of the sensitivity vector of the interferometer, the peak width is inversely proportional to the length $M$ of the sampling window used and the peak intensity depends on the amplitude of modulation of the intensity signal $I_{m}$ inside the sampling window.

When $k_{r}<4 / M$, interference effects may occur at $k_{r}$ due to a superposition with the negative frequency term (see Fig. 1). This also may occur near the Nyquist frequency limit at $k_{t}=2$, for $k_{r}>2-4 / M$. Therefore, the phase gradient $\dot{\Phi}_{d}$ will be restricted to the range $\left|\dot{\Phi}_{d}\right|<(1-4 / M) \phi \mathrm{rad}_{\text {frame }}{ }^{-1}$. As a result, the out-of-plane velocity of the sample (in $\mu \mathrm{m}$

frame $^{-1}$ ) will be restricted to the range:

$$
|\dot{z}|<\left(1-\frac{4}{M}\right) \frac{\lambda \phi}{4 \pi}
$$

For $M=64, \lambda=0.532 \mu \mathrm{m}, \phi=\pi / 2$ and $T=1 \mathrm{~ms}$, the velocity range is thus $|\dot{z}|<62.3 \mu \mathrm{m} \mathrm{s}^{-1}$, very close to the Nyquist limit $\left|\dot{z}_{N q}\right|=66.5 \mu \mathrm{m} \mathrm{s}^{-1}$. Eq. (30) shows that the allowed velocity range is greatly reduced for $M<8$. 
Short window TPS algorithms, which are associated with a broad peak in the frequency domain, ensure a non-zero signal level along the line $k_{t}=1$ even for large frequency shifts. On the contrary, when long-window TPS algorithms are used, the narrow peak of the transform in the frequency domain leaves the carrier frequency with a signal level too low and close to the noise level for phase evaluation. Such shifts may occur due to highamplitude low-frequency vibration of the test object and also to rigid body motion or low speed deformations during acquisition. As a result, the continuity of the measurement may be lost due to the absence of signal at $k_{t}=1$. A frequency shift greater than the peak halfwidth is interpreted in the time domain as the absence of the carrier frequency in the modulated intensity signal. It is easy to understand that under these circumstances, TPS algorithms which evaluate the phase of this carrier will fail.

Long window TPS algorithms are particularly useful when measurements are carried out in non-controlled environments and the presence of vibration could affect the precision of the measurements. Even though long window TPS algorithms show reduced sensitivity to vibration as a consequence of the integration process that occurs during the window duration, they are vulnerable -as proved for harmonic vibration- to frequencies close to the dc term and to twice the frequency of the carrier, with sensitivity bands whose widths depend inversely on the window length of the TPS algorithm. ${ }^{14}$ The ridge-searching algorithm aims to solve this problem by adapting the phase evaluation to the instantaneous carrier frequency produced by object movement and the action of the phase shifting device.

\section{A Numerical Simulation}

Typical results are presented in Fig. 3 from one of the simulations described in section 3.1, for the case $\sigma_{\phi}=3 \pi / 2$ or $\sigma_{z}=3 \lambda / 8$. The intensity signal and a gray level representation of 
the WFT of the signal, $\left|\widetilde{I}\left(k_{r}, t\right)\right|$, in the $k_{t}$ vs. $t$ domain (using a 32-frame Hanning window), are shown in Figs. 3(a) and (b), respectively. The calculated ridge position, $k_{\mathrm{r}}(t)$, is plotted in Fig. 3(c), with the intensity modulation evaluated along the line $k_{\mathrm{t}}=1$ displayed in Fig. 3(d).

Fig. 3(e) shows the original input phase change $\Delta \Phi(t, 0)=\Phi(t)-\Phi(0)$ obtained through the simulation described in section 3.1 , for $\sigma_{\phi}=3 \pi / 2$ or $\sigma_{z}=3 \lambda / 8$. ). The unwrapped phase change evaluated through standard and ridge-searching 32-frame algorithms are also shown. This is a typical example of a successful phase evaluation for the ridge-searching algorithm and of an incorrect one for a standard TPS algorithm. The phase calculated at $k_{t}=$ 1 is not correctly evaluated and considerable offset errors occur along the time history of the phase. By looking at the behavior of $k_{r}(t), \Delta \Phi(t)$ and $I_{m}(t)$, it can be seen that these errors occur especially when the peak of the WFT, i.e. the ridge, shifts considerably from $k_{t}$ $=1$ near frames 60 and 160. The intensity signal shows a loss of modulation near these frames. By contrast, in the regions between frames 16-60, 65-160 and 180-280, the slope of the phase, which is proportional to surface velocity, that is evaluated at $k_{t}=1$ is nearly the same as that of the phase evaluated over the ridge.

In order to measure the probability of a successful phase estimation without loss of phase continuity, we evaluated the unwrapping success rate over 100 signals $I(t)$ similar to that shown in Fig. 3(a). Table 1 shows the results for the USR for different standard and ridgesearching TPS algorithms based on the Hanning window. It is seen that the USR increases considerably when the ridge-searching algorithm is used, no matter what length is chosen for the sampling window, except for $\sigma_{\phi} \geq 2 \pi$ and $M=64$, which is a particularly difficult case because of the high amplitude of the vibration. 
Table 2 shows the rms phase change error $\bar{\sigma}$ for different TPS algorithms that evaluate the phase over $k_{t}=1$ or over the ridge $k_{r}(t)$. It is seen that there is no substantial difference in the rms error when the phase is evaluated over $k_{t}=1$ or over the ridge, even when the length of the sampling window is changed.

\section{B Experimental}

As an application example, we used the ridge searching algorithm to measure sub-surface delaminations in a carbon composite material when subjected to vacuum loading. The measurements were carried out in the presence of mechanical vibration and without vibration isolation, using a high-speed dynamic speckle interferometer running at $1 \mathrm{kHz}$

(see Fig. 4). ${ }^{5}$ Figure 5 shows a cross-section through the unwrapped phase map, corresponding to the out-of-plane surface displacement along a line above the delamination, obtained using both a standard 64-frame Hanning window algorithm (which evaluates the phase at $k_{t}=1$ ) and a ridge-searching algorithm with the same sampling window. Smoothing of the numerator and denominator of Eq. (5) was carried out by convolving with a $3 \times 3$ kernel of equal values, using the temporal unwrapping algorithm 3 in Ref. [5]. The plots show the accumulated deformation with respect to the first frame of a sequence of 512 frames. The difference is dramatic: while the ridge-searching algorithm allows the measurement to be done, the standard algorithm completely fails during phase evaluation.

\section{Conclusions}

In this paper, we presented a novel method for temporal phase evaluation of data from a high-speed speckle interferometer The algorithm is based on a first order approximation of the object phase inside the used sampling window and on the windowed Fourier transform 
(WFT), and involves the evaluation of the phase at the instantaneous ridge frequency $k_{t}=$ $k_{r}(t)$ instead of at the normalised carrier frequency $k_{t}=1$. The algorithm is especially useful when used with long sampling windows $M \geq 16$, in which case it is robust against low frequency vibration and presents low rms spatial errors in the phase change estimator. The algorithm is able to track the phase during the time history of the deformation without losing the continuity of the measurement and with low rms error, which was not possible with standard TPS algorithms. Therefore, the temporal history of a slow deformation can be

obtained, even in the presence of random vibration and without vibration isolation during the acquisition of the interferograms.

\section{Acknowledgments}

The authors wish to thank Fundación Antorchas of Argentina and The British Council for the research award Project $N^{\circ} 13888-18$ to support P. D. Ruiz's visit to Loughborough University.

\section{Author contact information}

Dr. Pablo Daniel Ruiz

Wolfson School of Mechanical and Manufacturing Engineering

Loughborough University, Leicestershire, LE11 3TU UK

Direct Line: +44(0)1509 227592

Fax: +44(0) 1509223934

E-mail: P.D.Ruiz@lboro.ac.uk 


\section{References}

1. See, for example, P. K. Rastogi, ed., Digital Speckle Interferometry Pattern Interferometry and Related Techniques, (Wiley, Chichester, 2001).

2. D. W. Robinson and G. T. Reid, eds., Interferogram Analysis (Institute of Physics, Bristol, 1993).

3. I. Yamaguchi, J. Y. Liu, and J. Kato, "Active phase-shifting interferometers for shape and deformation measurements," Opt. Eng. 35, 2930-2937 (1996).

4. X. C. de Lega, "Processing of non-stationary interference patterns: adapted phaseshifting algorithms and wavelet analysis. Application to dynamic deformation measurements by holographic and speckle interferometry," Ph.D. Dissertation, Ecole Polytechnique Fédérale de Lausanne, Lausanne (1997).

5. J. M. Huntley, G. H. Kaufmann, and D. Kerr, "Phase-shifted dynamic speckle pattern interferometry at 1 kHz," Appl. Opt. 38, 6556-6563 (1999).

6. P. Haible, M. P. Kothiyal, and H. J. Tiziani, "Heterodyne temporal speckle-pattern interferometry," Appl. Opt. 39, 114-117 (2000).

7. J. M. Kilpatrick, A. J. Moore, J. S. Barton, J. D. C. Jones, M. Reeves, and C. Buckberry, "Measurement of complex surface deformation at audio acoustic frequencies by high-speed dynamic phase-stepped ESPI,” Opt. Lett. 25, 1068-1070 (2000).

8. J. M. Huntley and H. Saldner, "Temporal phase-unwrapping algorithm for automated interferogram analysis," Appl. Opt. 32, 3047-3052 (1993). 
9. J. Schwider, R. Burow, K. E. Elssner, J. Grzanna, R. Spolaczyk, and K. Merkel, "Digital wave-front measuring interferometry - some systematic error sources," Appl. Opt. 22, 3421-3432 (1983).

10. P. Hariharan, B. F. Oreb, and T. Eiju, "Digital phase-shifting interferometry: a simple error-compensating phase calculation algorithm," Appl. Opt. 26, 2504-2506 (1987).

11. Y. Surrel, "Design of algorithms for phase measurements by the use of phase-stepping," Appl. Opt. 35, 51-60 (1996).

12. J. M. Huntley, "Suppression of phase errors from vibration in phase-shifting interferometry," J. Opt. Soc. Am. A 15, 2233-2241 (1998).

13. P. D. Ruiz, J. M. Huntley, J. Shen, C. R. Coggrave and G. H. Kaufmann, "Vibrationinduced phase errors in high-speed phase-shifting speckle pattern interferometry," Appl. Opt. 40, 2117-2125 (2001).

14. P. D. Ruiz, J. M. Huntley, J. Shen, C. R. Coggrave and G. H. Kaufmann, "Effects of random vibration in high-speed phase-shifting speckle pattern interferometry," Appl. Opt. 41, 3941-3949 (2002).

15. P. Carré, "Installation et utilisation du comparateur photoélectrique et interférentiel du Bureau International des Poids et Mesures", Metrologia 2, 13-23 (1966).

16. M. Cherbuliez , P. M. Jacquot and X. Colonna de Lega, "Wavelet processing of interferometric signals and fringe patterns", Proc. SPIE 3813, p. 692-702, Wavelet Applications in Signal and Image ProcessingVII, M. A. Unser, A. Aldroubi, and A. F. Laine, Eds (1999).

17. B. Bessason, C. Madshus, H. A. Frøystein and H. Kolbjørnsen, "Vibration criteria for metrology laboratories”, Meas. Sci. Technol. 10, 1009-1014 (1999). 
18. E. E. Ungar, D. H. Sturz and C. H. Amick, "Vibration Control Design of High Technology Facilities", Sound and Vibration, 7, 20-27 (1990). 


\section{Figure Captions}

Figure 1. Schematic view of the windowed Fourier transform of the intensity signal at an arbitrary time $t$ for which the ridge appears at $k_{r}(t)$. The transform of the 16-frame Hanning window is shown centered on each delta function. This case corresponds to an object moving with velocity $\dot{z}=\frac{\lambda}{8}\left(k_{r}-1\right) \mu \mathrm{m} \mathrm{s}^{-1}$ and the effect of the velocity is to shift the peak from $k_{t}=1$ to $k_{t}=k_{r}(t)$.

Figure 2. Velocity spectrum of the pseudo-random vibrations used in the numerical simulations.

Figure 3. Result of the analysis of a pseudo-random vibration signal having a spectrum similar to that shown in Fig. 2. (a) Intensity signal; (b) gray scale representation of the WFT of $I(t)$ in the $k_{t}$ vs. $t$ domain measured with the ridge-searching algorithm; (c) ridge trajectory $k_{r}(t)$; (d) intensity signal modulation $I_{m}$; (e) original input phase compared to the unwrapped phase change evaluated through standard (S-TPS) and ridge-searching (RSTPS) algorithms. In both cases a 32-frame Hanning window was used.

Figure 4. Dynamic high-speed phase-shifting speckle interferometer showing a Pockels cell in the reference beam $(P)$, high voltage driver $(D)$, function generator $(\mathrm{G})$, frame store (F), Sun computer (Sun), carbon composite laminate (O), Vacuum chamber (VC), 90:10 beam splitters (BS), mirrors (M) and lenses (L). Details on the synchronization of the system's electronics are given in Ref. (5). Internal delaminations in the composite material are revealed when it is vacuum loaded.

Figure 5. Phase profile measured from the surface of a carbon-fibre composite panel containing a sub-surface delamination, in the presence of mechanical vibration without 
vibration isolation, using 64-frame Hanning window algorithms. Phase evaluated over $k_{t}=$ 1 (S-TPS) and over the ridge $k_{r}(t)$ (RS-TPS). 
Table 1. Unwrapping success rate numerical results.

\begin{tabular}{cccccc}
\hline$\sigma_{\phi}(\mathrm{rad})$ & $k_{t}$ & $\mathrm{M}=8$ & $\mathrm{M}=16$ & $\mathrm{M}=32$ & $\mathrm{M}=64$ \\
\hline \multirow{2}{*}{$2 \pi$} & 1 & 0.93 & 0.40 & 0.21 & 0.18 \\
\cline { 2 - 6 } & $k_{r}(t)$ & 1 & 0.99 & 0.67 & 0.15 \\
\hline \multirow{2}{*}{$3 \pi / 2$} & 1 & 0.99 & 0.66 & 0.29 & 0.23 \\
\cline { 2 - 6 } & $k_{r}(t)$ & 1 & 0.99 & 0.87 & 0.30 \\
\hline \multirow{2}{*}{$\pi$} & 1 & 1 & 0.98 & 0.76 & 0.46 \\
\cline { 2 - 6 } & $k_{r}(t)$ & 1 & 1 & 1 & 0.71 \\
\hline
\end{tabular}

The USR is evaluated for different vibration phase rms amplitudes $\sigma_{\phi}$, using TPS algorithms that evaluate the phase over $k_{t}=1$ or over the ridge $k_{r}(t)$. In all cases, $M$-frame Hanning sampling windows and a camera framing rate of $1 \mathrm{kHz}$ were used. 
Table 2. Numerical results of the rms phase change error $\bar{\sigma}$.

\begin{tabular}{|c|c|c|c|c|}
\hline \multicolumn{2}{|c|}{ Algorithm } & \multicolumn{3}{|c|}{ Framing Rate $(\mathrm{kHz})$} \\
\hline $\mathrm{M}$ & $k_{t}$ & 1 & 2 & 4 \\
\hline \multirow{2}{*}{8} & 1 & 0.02215 & 0.02107 & 0.02077 \\
\hline & $k_{r}(t)$ & 0.02022 & 0.01637 & 0.01460 \\
\hline \multirow{2}{*}{16} & 1 & 0.00351 & 0.00271 & 0.00262 \\
\hline & $k_{r}(t)$ & 0.00265 & 0.00170 & 0.00145 \\
\hline \multirow{2}{*}{32} & 1 & 0.00136 & 0.00036 & 0.00033 \\
\hline & $k_{r}(t)$ & 0.00126 & 0.00022 & 0.00020 \\
\hline \multirow{2}{*}{64} & 1 & 0.00108 & --- & --- \\
\hline & $k_{r}(t)$ & 0.00091 & --- & --- \\
\hline
\end{tabular}

Values of $\bar{\sigma}(\mathrm{rad})$ obtained with different TPS algorithms based on the $M$-frame Hanning window, that evaluate the phase over $k_{t}=1$ or over the ridge $k_{t}=k_{r}(t)$. The values were obtained using simulated data with an input phase rms error $\sigma_{\phi}=\pi \mathrm{rad}$. 


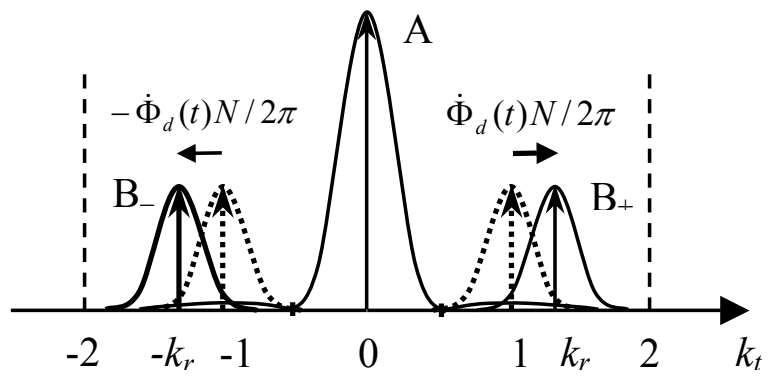

Fig. (1) Ruiz et al

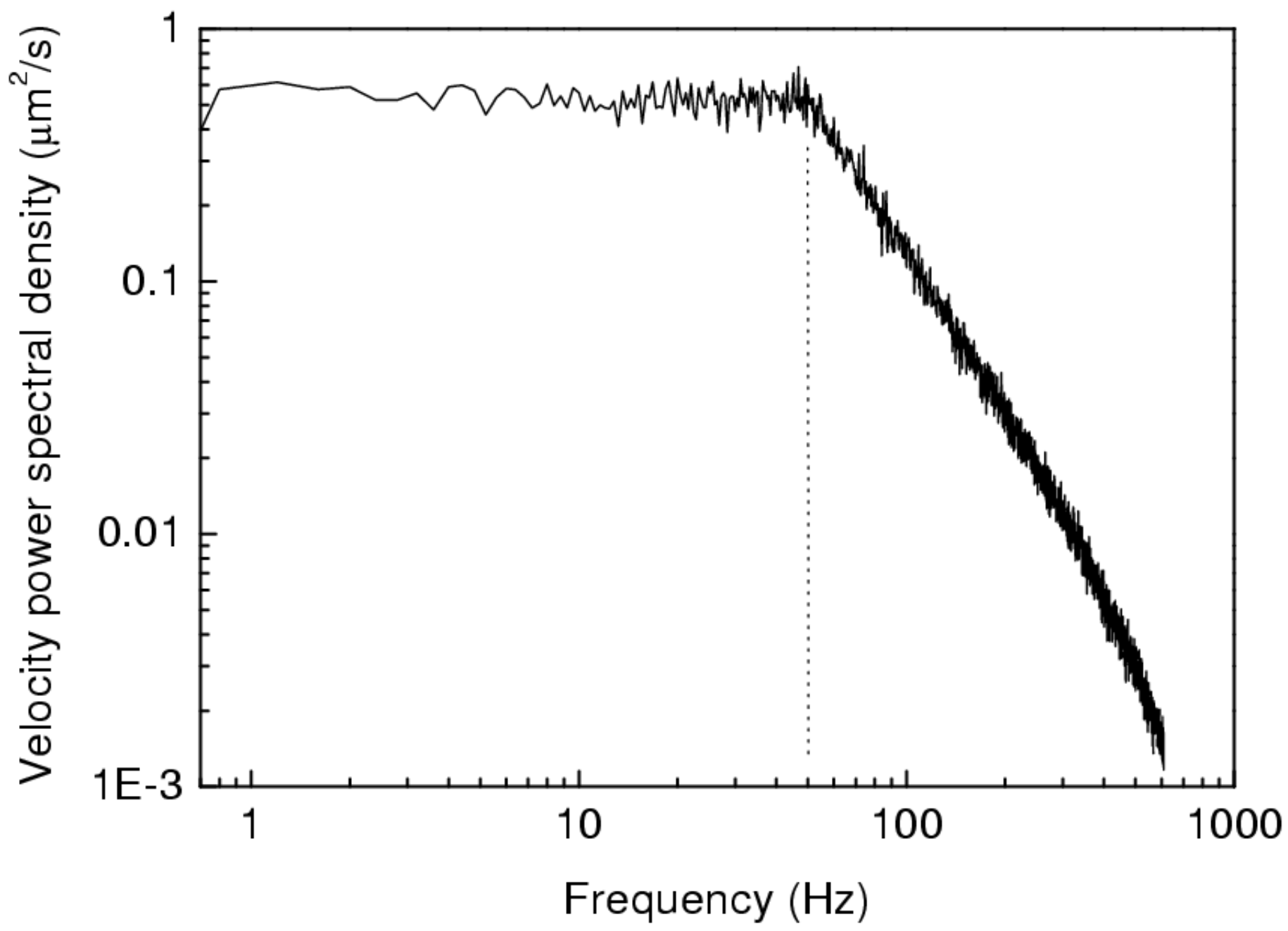

Fig. (2) Ruiz et al 


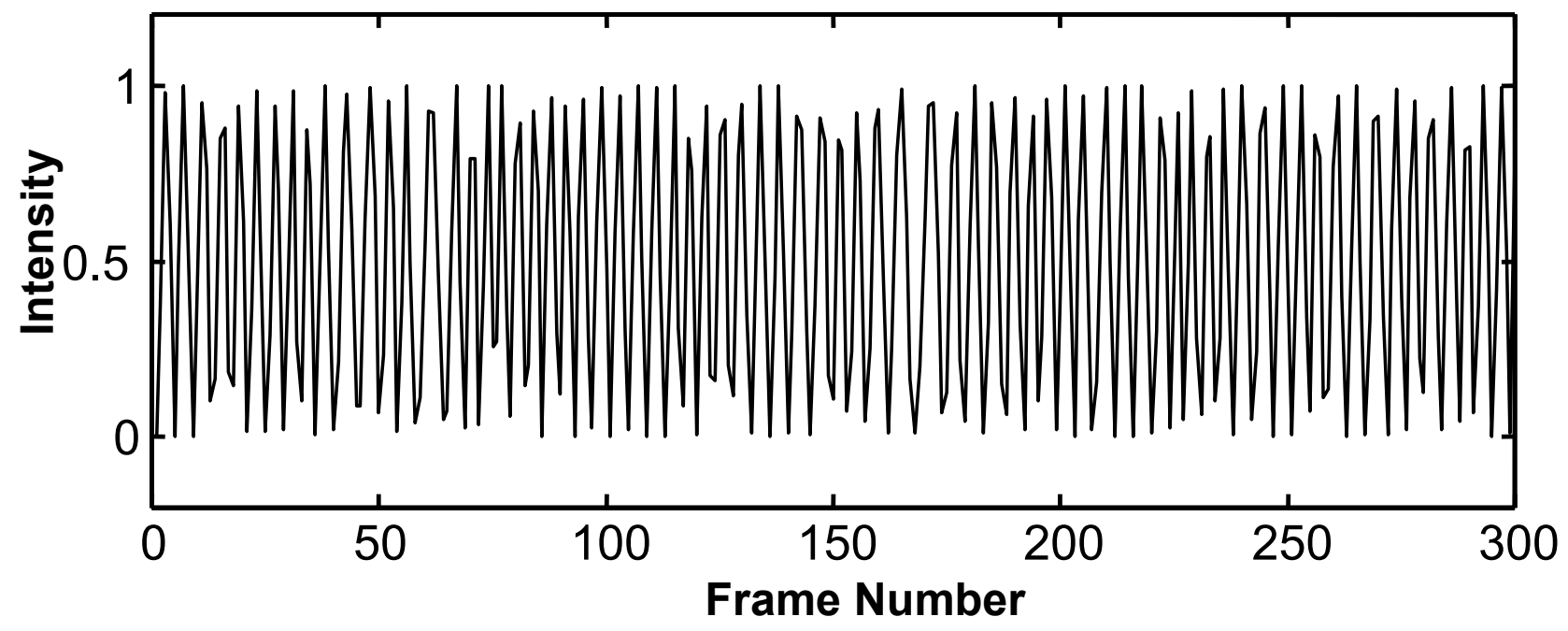

Fig. 3(a) Ruiz et al

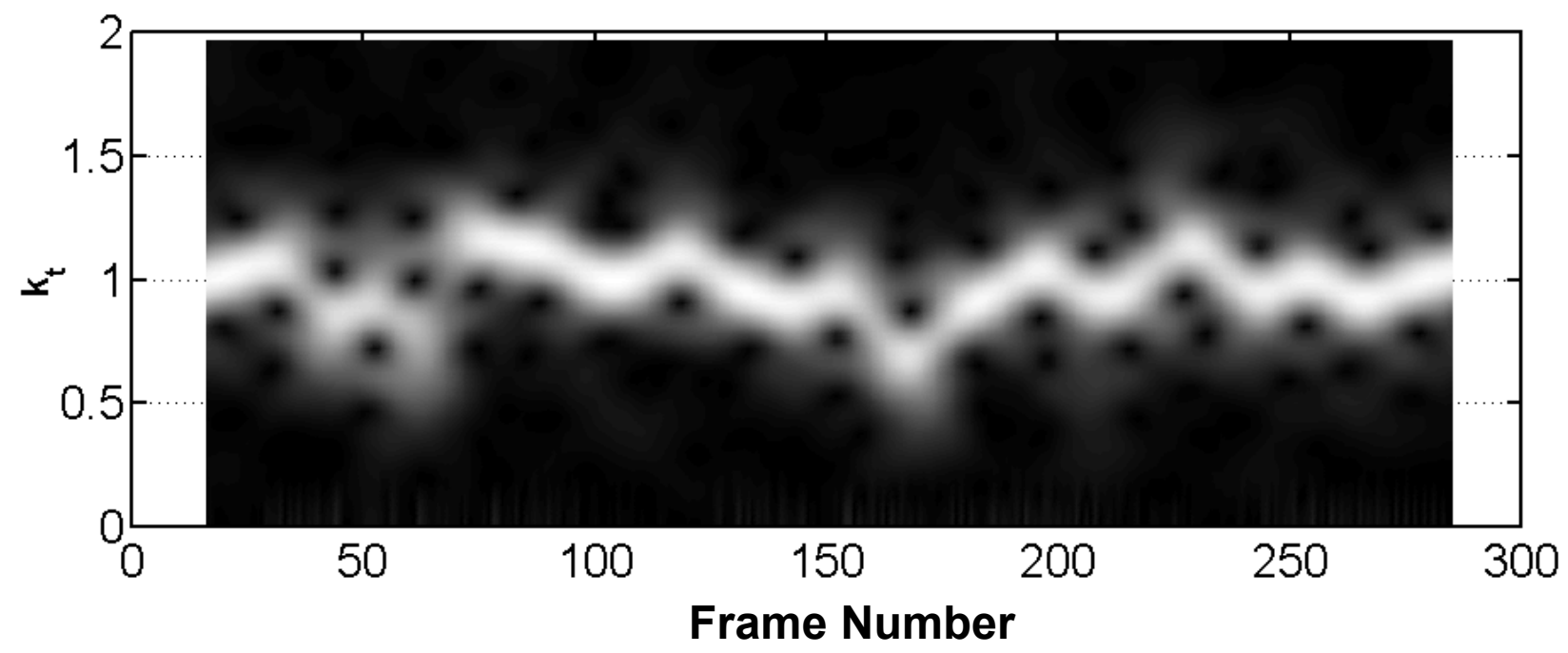

Fig. 3(b) Ruiz et al 


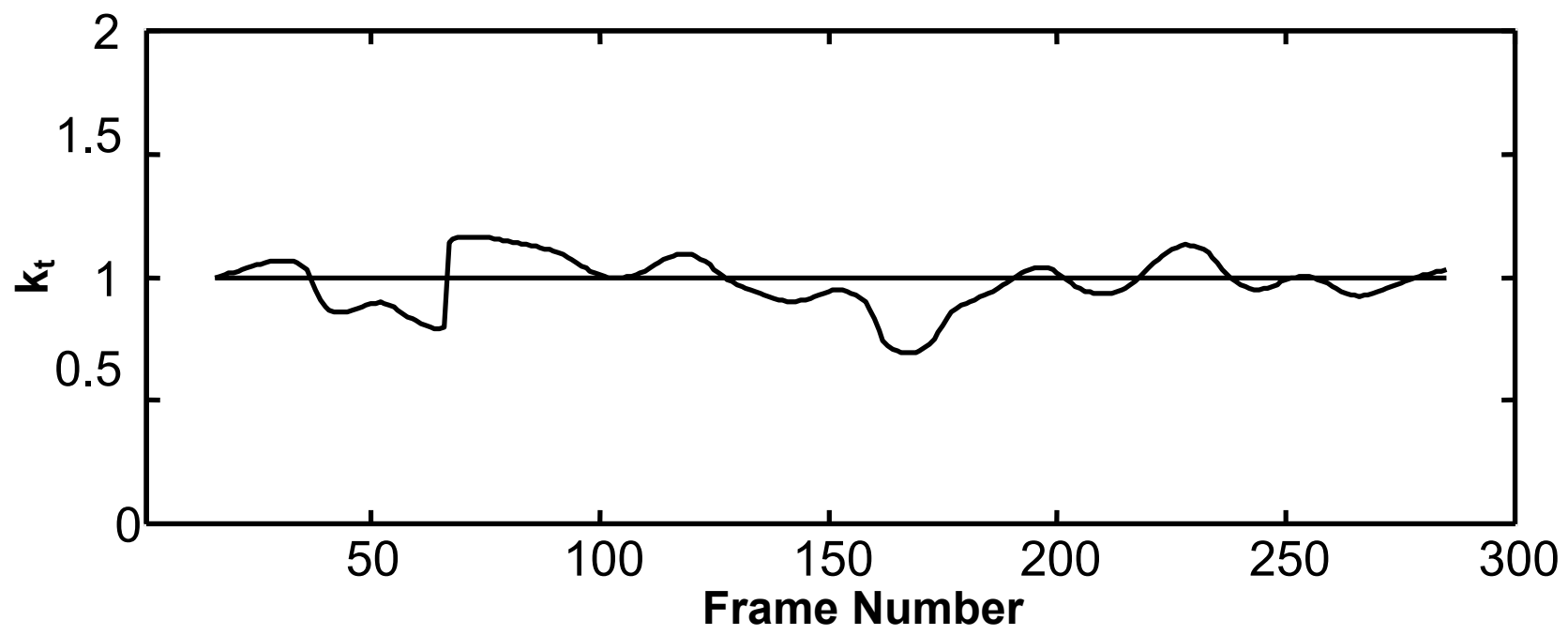

Fig. 3(c) Ruiz et al

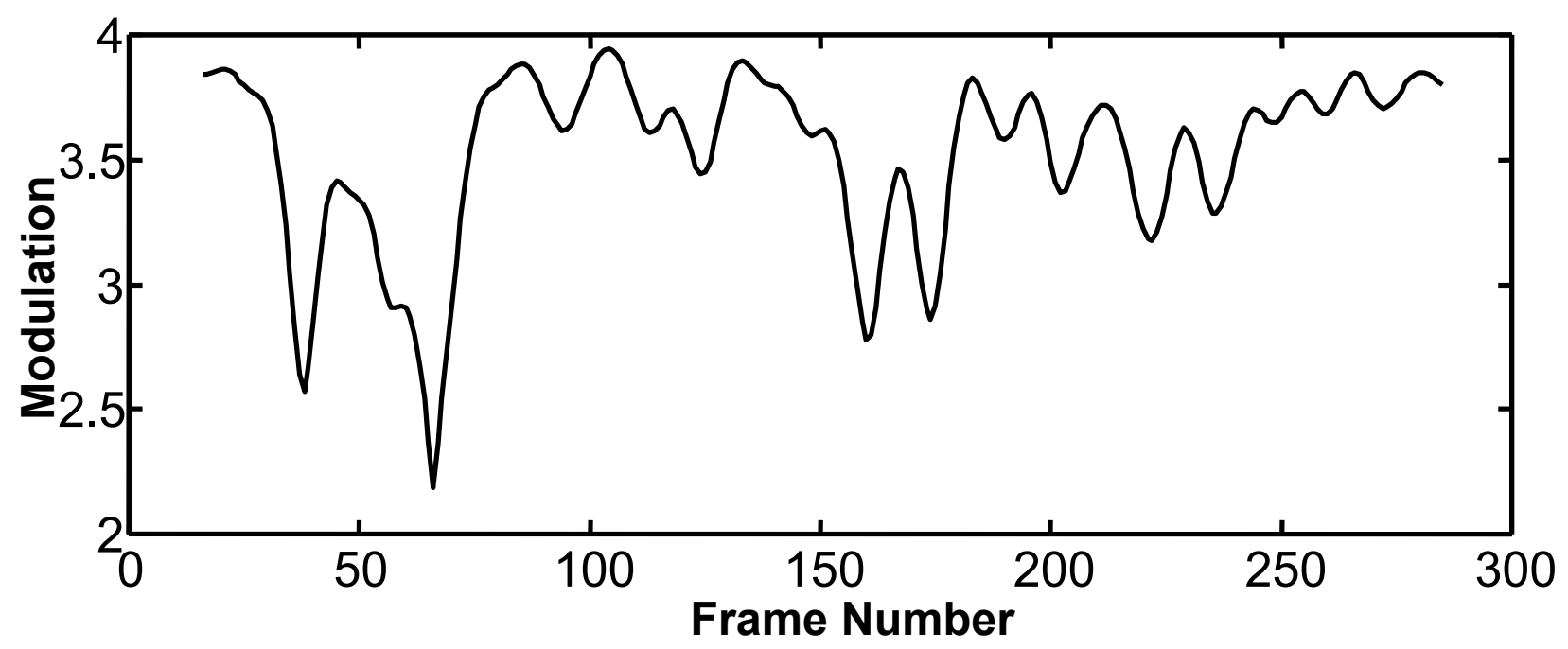

Fig.3 (d) Ruiz et al 


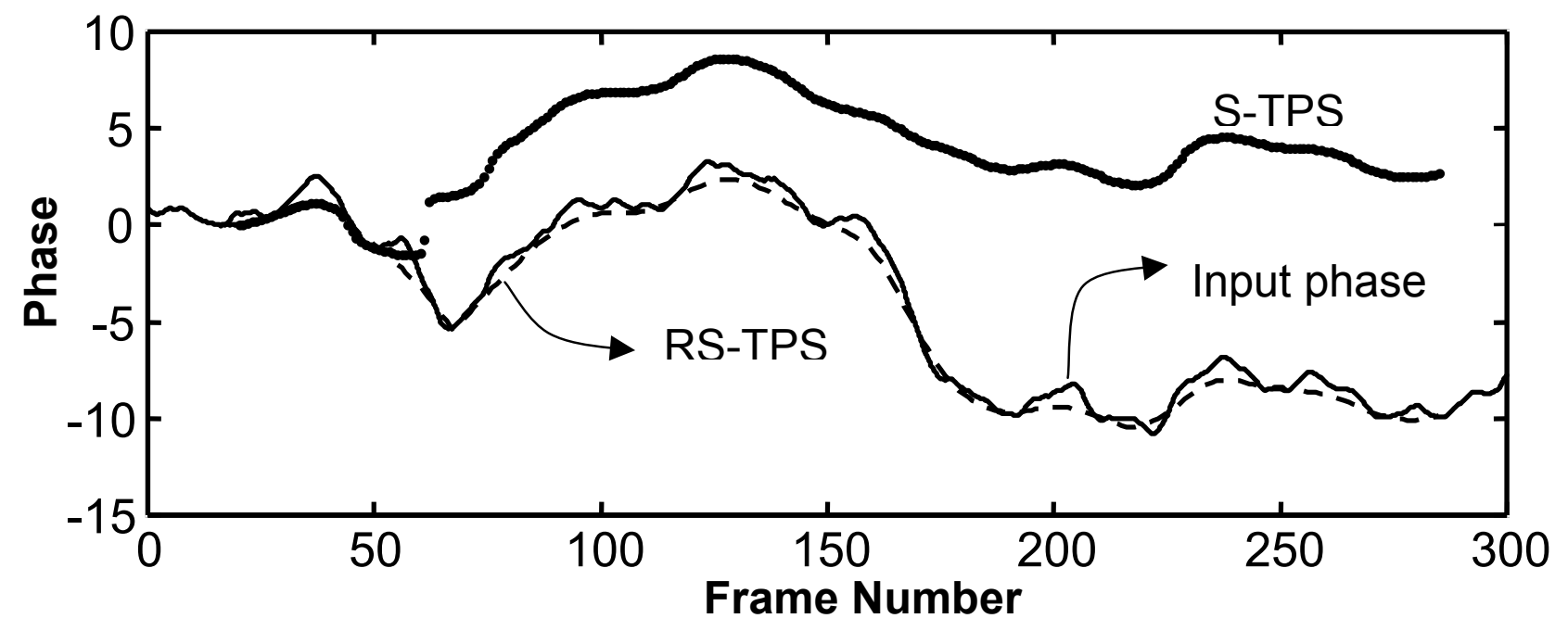

Fig. 3 (e) Ruiz et al

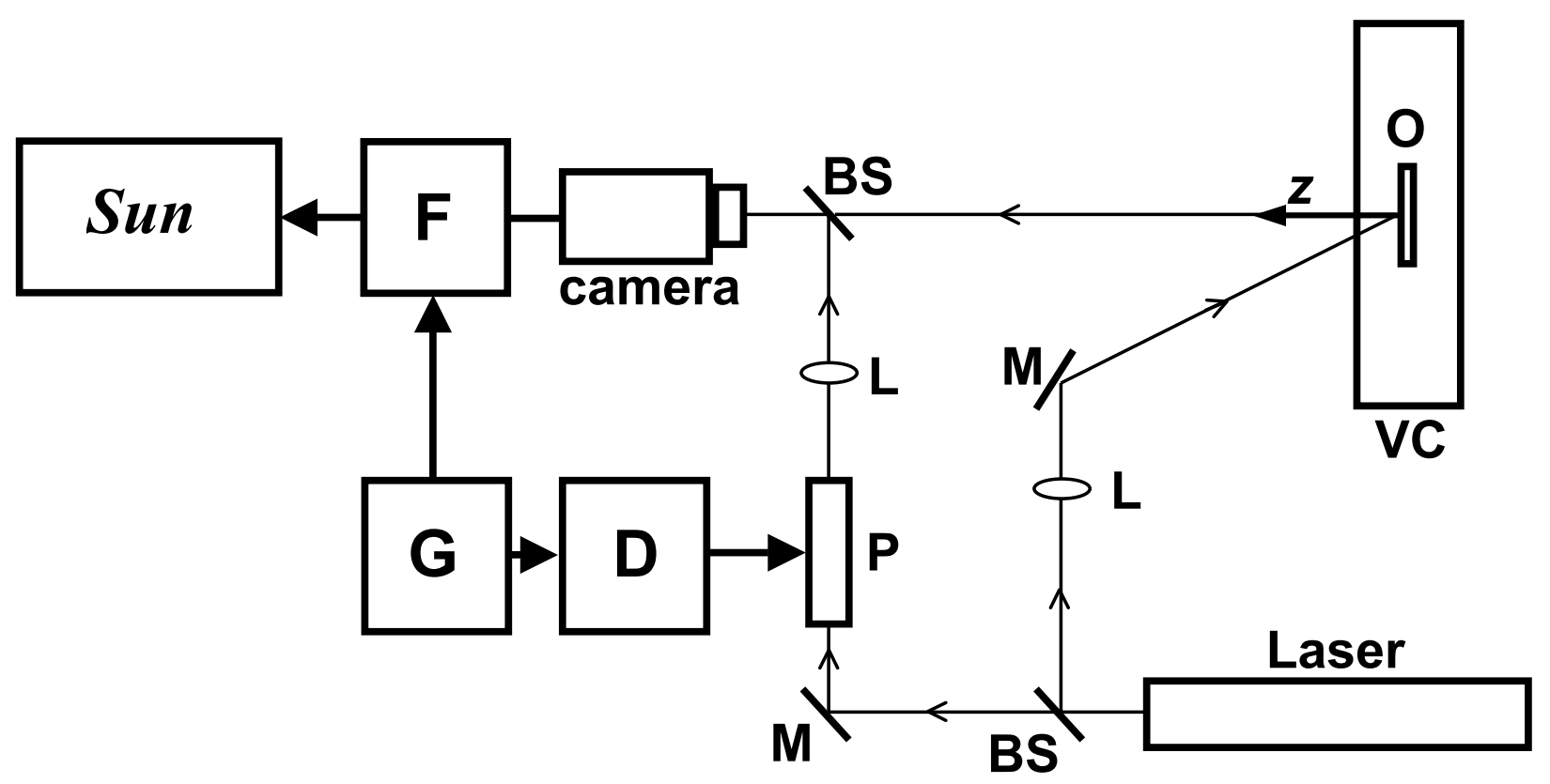

Fig. 4 Ruiz et al 


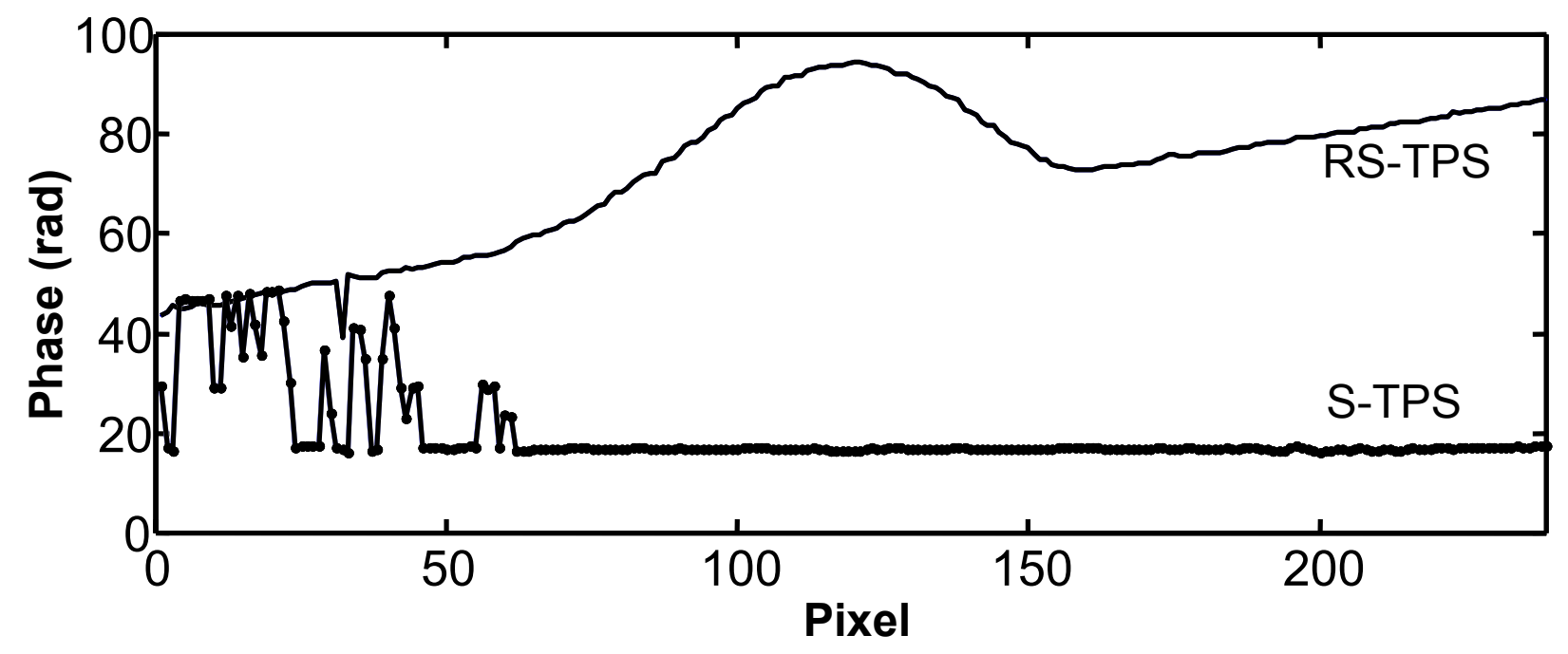

Fig. 5 Ruiz et al 